\title{
2016 Audited schedule of changes in net assets
}

\section{Ray Naegele}

See end of article for authors' affiliations.

The table below summarizes the association's financial status as of December 31, 2016. For a more complete audit report and related information, see the 2015/16 MLA Annual Report (members only). This report includes balance sheets, fund status reports, budgeted and actual revenues and expenditures, and a schedule of investments.

Members may obtain a copy of the audit report from MLA headquarters.

Additional information regarding MLA finances appears in the May 2017 issue of the MLA News.

Table 1 Medical Library Association schedule of changes in net assets by fund year ended December 31, 2016

\begin{tabular}{|c|c|c|c|c|c|c|}
\hline & $\begin{array}{c}\text { Net assets } \\
\text { January 1, } \\
2016\end{array}$ & $\begin{array}{c}\text { Contributions } \\
\text { and other } \\
\text { revenue }\end{array}$ & $\begin{array}{c}\text { Invest- } \\
\text { ment } \\
\text { income }\end{array}$ & $\begin{array}{l}\text { Net assets } \\
\text { released } \\
\text { from } \\
\text { restrictions }\end{array}$ & Expenses & $\begin{array}{c}\text { Net assets } \\
\text { December } \\
31,2016 \\
\end{array}$ \\
\hline \multicolumn{7}{|l|}{ Unrestricted net assets } \\
\hline General operating & $(\$ 223,468)$ & $\$ 2,763,212$ & - & - & $(\$ 2,748,542)$ & $(\$ 208,798)$ \\
\hline \multicolumn{7}{|l|}{ Other funds: } \\
\hline Association stabilization & $\$ 1,448,483$ & - & $\$ 83,893$ & - & - & $\$ 1,532,376$ \\
\hline Capital equipment & $\$ 2,228$ & - & - & - & - & $\$ 2,228$ \\
\hline Special purpose & - & $\$ 33,096$ & - & $\$ 128,983$ & $(\$ 128,983)$ & $\$ 33,096$ \\
\hline Sections & $\$ 364,336$ & $\$ 49,299$ & - & - & $(\$ 132,572)$ & $\$ 281,063$ \\
\hline $\begin{array}{l}\text { Total unrestricted net } \\
\text { assets }\end{array}$ & $\$ 1,815,047$ & $\$ 82,395$ & $\$ 83,893$ & $\$ 128,983$ & $(\$ 261,555)$ & $\$ 1,848,763$ \\
\hline \multicolumn{7}{|l|}{$\begin{array}{l}\text { Temporarily restricted } \\
\text { net assets }\end{array}$} \\
\hline $\begin{array}{l}\text { Ysabel Bertolucci MLA } \\
\text { Annual Meeting Grant } \\
\text { Endowment }\end{array}$ & $(\$ 554)$ & - & $\$ 1,604$ & $(\$ 1,000)$ & - & $\$ 50$ \\
\hline $\begin{array}{l}\text { Estelle Brodman Award } \\
\text { for the Academic } \\
\text { Medical Librarian of the } \\
\text { Year Endowment }\end{array}$ & $\$ 39,834$ & - & $\$ 1,746$ & $(\$ 500)$ & - & $\$ 41,080$ \\
\hline $\begin{array}{l}\text { Naomi C. Broering } \\
\text { Hispanic Heritage Grant } \\
\text { Endowment }\end{array}$ & $(\$ 516)$ & - & $\$ 1,522$ & - & - & $\$ 1,006$ \\
\hline $\begin{array}{l}\text { Lois Ann Colaianni } \\
\text { Award for Excellence } \\
\text { and Achievement in } \\
\text { Hospital Librarianship } \\
\text { Endowment }\end{array}$ & $\$ 9,157$ & - & $\$ 912$ & $(\$ 500)$ & - & $\$ 9,569$ \\
\hline
\end{tabular}


DOI: dx.doi.org/10.5195/jmla.2017.340

Table 1 Medical Library Association schedule of changes in net assets by fund year ended December 31, 2016 (continued)

\begin{tabular}{|c|c|c|c|c|c|c|}
\hline & $\begin{array}{c}\text { Net assets } \\
\text { January 1, } \\
2016 \\
\end{array}$ & $\begin{array}{c}\text { Contributions } \\
\text { and other } \\
\text { revenue }\end{array}$ & $\begin{array}{l}\text { Invest- } \\
\text { ment } \\
\text { income }\end{array}$ & $\begin{array}{l}\text { Net assets } \\
\text { released } \\
\text { from } \\
\text { restrictions }\end{array}$ & Expenses & $\begin{array}{c}\text { Net assets } \\
\text { December } \\
31,2016 \\
\end{array}$ \\
\hline $\begin{array}{l}\text { Cunningham Memorial } \\
\text { International Fellowship } \\
\text { Endowment }\end{array}$ & $\$ 26,685$ & - & $\$ 7,344$ & $(\$ 4,104)$ & - & $\$ 29,925$ \\
\hline $\begin{array}{l}\text { Louise Darling Medal } \\
\text { for Distinguished } \\
\text { Achievement in } \\
\text { Collection Development } \\
\text { in the Health Sciences } \\
\text { Endowment }\end{array}$ & $\$ 40,576$ & - & $\$ 2,159$ & - & - & $\$ 42,735$ \\
\hline $\begin{array}{l}\text { Janet Doe Lectureship } \\
\text { Endowment }\end{array}$ & $\$ 29,429$ & - & $\$ 1,544$ & (\$811) & - & $\$ 30,162$ \\
\hline $\begin{array}{l}\text { Carla J. Funk } \\
\text { Governmental Relations } \\
\text { Award Endowment }\end{array}$ & $\$ 795$ & - & $\$ 844$ & - & - & $\$ 1,639$ \\
\hline $\begin{array}{l}\text { Eugene Garfield } \\
\text { Research Fellowship } \\
\text { Endowment }\end{array}$ & $(\$ 5,876)$ & - & $\$ 7,468$ & $(\$ 5,000)$ & - & $(\$ 3,408)$ \\
\hline $\begin{array}{l}\text { T. Mark Hodges } \\
\text { International Service } \\
\text { Award Endowment }\end{array}$ & $(\$ 61)$ & - & $\$ 287$ & - & - & $\$ 226$ \\
\hline $\begin{array}{l}\text { Hospital Libraries } \\
\text { Section/MLA } \\
\text { Professional } \\
\text { Development Grant } \\
\text { Endowment }\end{array}$ & $\$ 18,024$ & - & $\$ 1,652$ & $(\$ 1,589)$ & - & $\$ 18,087$ \\
\hline $\begin{array}{l}\text { David A. Kronick } \\
\text { Traveling Fellowship } \\
\text { Endowment }\end{array}$ & $(\$ 1,307)$ & - & $\$ 1,581$ & $(\$ 1,986)$ & - & $(\$ 1,712)$ \\
\hline $\begin{array}{l}\text { Joseph Leiter } \\
\text { NLM/MLA Lectureship } \\
\text { Endowment }\end{array}$ & $\$ 28,317$ & - & $\$ 2,467$ & - & - & $\$ 30,784$ \\
\hline $\begin{array}{l}\text { Librarians without } \\
\text { Borders® Ursula Poland } \\
\text { International } \\
\text { Scholarship Endowment }\end{array}$ & $\$ 1,793$ & - & $\$ 1,344$ & - & - & $\$ 3,137$ \\
\hline $\begin{array}{l}\text { Donald A. B. Lindberg } \\
\text { Research Fellowship } \\
\text { Endowment }\end{array}$ & $\$ 67,000$ & $\$ 5,351$ & $\$ 12,606$ & $(\$ 20,000)$ & - & $\$ 64,957$ \\
\hline $\begin{array}{l}\text { Majors/MLA Chapter } \\
\text { Project of the Year } \\
\text { Endowment }\end{array}$ & $\$ 6,982$ & - & $\$ 687$ & $(\$ 500)$ & - & $\$ 7,169$ \\
\hline $\begin{array}{l}\text { Lucretia W. McClure } \\
\text { MLA Excellence in } \\
\text { Education Award } \\
\text { Endowment }\end{array}$ & $\$ 11,266$ & - & $\$ 2,068$ & $(\$ 500)$ & - & $\$ 12,834$ \\
\hline
\end{tabular}


Table 1 Medical Library Association schedule of changes in net assets by fund year ended December 31, 2016 (continued)

\begin{tabular}{|c|c|c|c|c|c|c|}
\hline & $\begin{array}{c}\text { Net assets } \\
\text { January 1, } \\
2016\end{array}$ & $\begin{array}{l}\text { Contributions } \\
\text { and other } \\
\text { revenue }\end{array}$ & $\begin{array}{c}\text { Invest- } \\
\text { ment } \\
\text { income }\end{array}$ & $\begin{array}{l}\text { Net assets } \\
\text { released } \\
\text { from } \\
\text { restrictions }\end{array}$ & Expenses & $\begin{array}{c}\text { Net assets } \\
\text { December } \\
31,2016\end{array}$ \\
\hline $\begin{array}{l}\text { John P. McGovern } \\
\text { Award Lectureship } \\
\text { Endowment }\end{array}$ & $(\$ 246)$ & - & $\$ 6,900$ & $(\$ 5,000)$ & - & $\$ 1,654$ \\
\hline $\begin{array}{l}\text { MLA Disaster Relief } \\
\text { Fund }\end{array}$ & $\$ 5,404$ & $\$ 850$ & - & - & - & $\$ 6,254$ \\
\hline Scholarship Endowment & $(\$ 12,941)$ & - & $\$ 15,501$ & $(\$ 14,962)$ & - & $(\$ 12,402)$ \\
\hline $\begin{array}{l}\text { Section Project of the } \\
\text { Year Award } \\
\text { Endowment }\end{array}$ & $\$ 858$ & - & - & $(\$ 500)$ & - & $\$ 358$ \\
\hline $\begin{array}{l}\text { Shaping Our Future } \\
\text { Endowment }\end{array}$ & $\$ 8,598$ & - & $\$ 2,881$ & - & - & $\$ 11,479$ \\
\hline $\begin{array}{l}\text { Special } \\
\text { Purpose/Librarians } \\
\text { without Borders® }\end{array}$ & $\$ 22,211$ & $\$ 135,005$ & - & $(\$ 46,031)$ & - & $\$ 111,185$ \\
\hline $\begin{array}{l}\text { Special } \\
\text { Purpose/MLA/NLM } \\
\text { Spectrum Scholarships }\end{array}$ & $\$ 65,000$ & - & - & $(\$ 26,000)$ & - & $\$ 39,000$ \\
\hline $\begin{array}{l}\text { Total, temporarily } \\
\text { restricted net assets }\end{array}$ & $\$ 360,428$ & $\$ 141,206$ & $\$ 73,117$ & $(\$ 128,983)$ & - & $\$ 445,768$ \\
\hline \multicolumn{7}{|l|}{$\begin{array}{l}\text { Permanently restricted } \\
\text { net assets: }\end{array}$} \\
\hline $\begin{array}{l}\text { Ysabel Bertolucci MLA } \\
\text { Annual Meeting Grant } \\
\text { Endowment }\end{array}$ & $\$ 26,868$ & $\$ 1,976$ & - & - & - & $\$ 28,844$ \\
\hline $\begin{array}{l}\text { Estelle Brodman Award } \\
\text { for the Academic } \\
\text { Medical Librarian of the } \\
\text { Year Endowment }\end{array}$ & $\$ 29,248$ & $\$ 250$ & - & - & - & $\$ 29,498$ \\
\hline $\begin{array}{l}\text { Naomi C. Broering } \\
\text { Hispanic Heritage Grant } \\
\text { Endowment }\end{array}$ & $\$ 25,493$ & $\$ 15$ & - & - & - & $\$ 25,508$ \\
\hline $\begin{array}{l}\text { Lois Ann Colaianni } \\
\text { Award for Excellence } \\
\text { and Achievement in } \\
\text { Hospital Librarianship } \\
\text { Endowment }\end{array}$ & $\$ 15,275$ & - & - & - & - & $\$ 15,275$ \\
\hline $\begin{array}{l}\text { Consumer Health } \\
\text { Librarian of the Year } \\
\text { Award Endowment }\end{array}$ & - & $\$ 12,584$ & - & - & - & $\$ 12,584$ \\
\hline $\begin{array}{l}\text { Cunningham Memorial } \\
\text { International Fellowship } \\
\text { Endowment }\end{array}$ & $\$ 123,021$ & $\$ 50$ & - & - & - & $\$ 123,071$ \\
\hline
\end{tabular}


DOI: dx.doi.org/10.5195/jmla.2017.340

Table 1 Medical Library Association schedule of changes in net assets by fund year ended December 31, 2016 (continued)

\begin{tabular}{|c|c|c|c|c|c|c|}
\hline & $\begin{array}{c}\text { Net assets } \\
\text { January 1, } \\
2016\end{array}$ & $\begin{array}{c}\text { Contributions } \\
\text { and other } \\
\text { revenue }\end{array}$ & $\begin{array}{c}\text { Invest- } \\
\text { ment } \\
\text { income }\end{array}$ & $\begin{array}{l}\text { Net assets } \\
\text { released } \\
\text { from } \\
\text { restrictions }\end{array}$ & Expenses & $\begin{array}{c}\text { Net assets } \\
\text { December } \\
31,2016\end{array}$ \\
\hline $\begin{array}{l}\text { Louise Darling Medal } \\
\text { for Distinguished } \\
\text { Achievement in } \\
\text { Collection Development } \\
\text { in the Health Sciences } \\
\text { Endowment }\end{array}$ & $\$ 36,168$ & - & - & - & - & $\$ 36,168$ \\
\hline $\begin{array}{l}\text { Janet Doe Lectureship } \\
\text { Endowment }\end{array}$ & $\$ 25,859$ & $\$ 200$ & - & - & - & $\$ 26,059$ \\
\hline $\begin{array}{l}\text { Carla J. Funk } \\
\text { Governmental Relations } \\
\text { Award Endowment }\end{array}$ & $\$ 14,140$ & $\$ 1,213$ & - & - & - & $\$ 15,353$ \\
\hline $\begin{array}{l}\text { Eugene Garfield } \\
\text { Research Fellowship } \\
\text { Endowment }\end{array}$ & $\$ 125,101$ & - & - & - & - & $\$ 125,101$ \\
\hline $\begin{array}{l}\text { T. Mark Hodges } \\
\text { International Service } \\
\text { Award Endowment }\end{array}$ & $\$ 4,815$ & $\$ 50$ & - & - & - & $\$ 4,865$ \\
\hline $\begin{array}{l}\text { Hospital Libraries } \\
\text { Section/MLA } \\
\text { Professional } \\
\text { Development Grant } \\
\text { Endowment }\end{array}$ & $\$ 27,680$ & $\$ 101$ & - & - & - & $\$ 27,781$ \\
\hline $\begin{array}{l}\text { David A. Kronick } \\
\text { Traveling Fellowship } \\
\text { Endowment }\end{array}$ & $\$ 26,487$ & $\$ 503$ & - & - & - & $\$ 26,990$ \\
\hline $\begin{array}{l}\text { Joseph Leiter } \\
\text { NLM/MLA Lectureship } \\
\text { Endowment }\end{array}$ & $\$ 41,328$ & - & - & - & - & $\$ 41,328$ \\
\hline $\begin{array}{l}\text { Librarians without } \\
\text { Borders® Ursula Poland } \\
\text { International } \\
\text { Scholarship Endowment }\end{array}$ & $\$ 22,515$ & $\$ 150$ & - & - & - & $\$ 22,665$ \\
\hline $\begin{array}{l}\text { Donald A. B. Lindberg } \\
\text { Research Fellowship } \\
\text { Endowment }\end{array}$ & $\$ 211,162$ & $\$ 5,962$ & - & - & - & $\$ 217,124$ \\
\hline $\begin{array}{l}\text { Majors/MLA Chapter } \\
\text { Project of the Year } \\
\text { Endowment }\end{array}$ & $\$ 11,500$ & - & - & - & - & $\$ 11,500$ \\
\hline $\begin{array}{l}\text { Lucretia W. McClure } \\
\text { MLA Excellence in } \\
\text { Education Award } \\
\text { Endowment }\end{array}$ & $\$ 34,634$ & $\$ 846$ & - & - & - & $\$ 35,480$ \\
\hline $\begin{array}{l}\text { John P. McGovern } \\
\text { Award Lectureship } \\
\text { Endowment }\end{array}$ & $\$ 115,585$ & - & - & - & - & $\$ 115,585$ \\
\hline
\end{tabular}


Table 1 Medical Library Association schedule of changes in net assets by fund year ended December 31, 2016 (continued)

\begin{tabular}{|c|c|c|c|c|c|c|}
\hline & $\begin{array}{c}\text { Net assets } \\
\text { January 1, } \\
2016\end{array}$ & $\begin{array}{l}\text { Contributions } \\
\text { and other } \\
\text { revenue }\end{array}$ & $\begin{array}{c}\text { Invest- } \\
\text { ment } \\
\text { income }\end{array}$ & $\begin{array}{l}\text { Net assets } \\
\text { released } \\
\text { from } \\
\text { restrictions }\end{array}$ & Expenses & $\begin{array}{c}\text { Net assets } \\
\text { December } \\
31,2016\end{array}$ \\
\hline Scholarship Endowment & $\$ 259,667$ & $\$ 4,287$ & - & - & - & $\$ 263,954$ \\
\hline $\begin{array}{l}\text { Shaping Our Future } \\
\text { Endowment }\end{array}$ & $\$ 48,269$ & $\$ 2,335$ & - & - & - & $\$ 50,604$ \\
\hline $\begin{array}{l}\text { Total, permanently } \\
\text { restricted net assets }\end{array}$ & $\$ 1,224,815$ & $\$ 30,522$ & - & - & - & $\$ 1,255,337$ \\
\hline Total all net assets & $\$ 3,176,822$ & $\$ 3,017,335$ & $\$ 157,010$ & \$- & $(\$ 3,010,097)$ & $\$ 3,341,070$ \\
\hline
\end{tabular}

\section{AUTHOR'S AFFILIATION}

Ray Naegele, naegele@mail.mlahq.org, Medical Library Association, 65 East Wacker Place, Suite 1900, Chicago, IL 60601-7246 